\title{
DETERMINATION OF VOLATILES IN WATER LILY FLOWERS USING GAS CHROMATOGRAPHY-MASS SPECTROMETRY
}

\author{
Ruyu Yuan ${ }^{\mathrm{a}, \mathrm{b}}$, Shanshan $\mathrm{Li}^{\mathrm{b}, \mathrm{c}}$, Xuchen Zheng ${ }^{\mathrm{a}, \mathrm{b}}$, Qian Wu ${ }^{\mathrm{a}, \mathrm{b}}$, Huijin Zhang ${ }^{\mathrm{b}}$, Liangsheng Wang ${ }^{\mathrm{b} *}$ \\ ${ }^{a}$ College of Horticulture, Nanjing Agricultural University, Nanjing 210095, China \\ ${ }^{\mathrm{b}}$ Beijing Botanical Garden/Key Laboratory of Plant Resources, Institute of Botany The Chinese \\ Academy of Sciences, Beijing 100093, China \\ ${ }^{c}$ University of Chinese Academy of Sciences, Beijing 100049, China
}

\section{SUPPLEMENTAL MATERIAL}


Supplemental Table 1. Types and concentrations (ng/g) of volatile compounds in hardy water lily flowers. Cultivars are coded as in Table 1

\begin{tabular}{|c|c|c|c|c|c|c|c|c|c|c|c|c|c|c|c|c|c|c|c|c|c|c|c|c|c|c|c|c|c|c|c|c|c|c|c|}
\hline compenans & $\overline{\mathrm{RT}(\text { min })}$ & ' & 2 & 3 & ${ }^{4}$ & s & 6 & ? & ${ }^{8}$ & , & ${ }_{10}^{10}$ & " & 12 & 13 & ${ }_{14}$ & is & ${ }_{16}^{16}$ & ${ }_{17}$ & 18 & 19 & 20 & ${ }^{21}$ & 22 & 2 & ${ }_{24}$ & 25 & 26 & 27 & 28 & 29 & 30 & ${ }_{31}$ & 32 & 33 & 3 \\
\hline 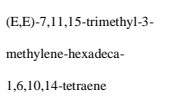 & ${ }^{43384}$ & 000 & 0.000 & 0.000 & $0 \infty$ & 000 & $8, s, 50005$ & 000 & 0000 & 0.00 & 0,0 & 0.00 & 0000 & oov & 0.00 & ${ }_{000}$ & 0.00 & 0.00 & 0.00 & ${ }_{527}$ & 0.000 & 0.00 & $\begin{array}{l}10060 \pm 00 \\
2\end{array}$ & 0.00 & 0.00 & 0.00 & $492 \pm 0008$ & $\infty$ & on & postoo & 000 & 000 & nom & 000 & 12320004 \\
\hline 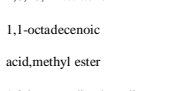 & ${ }_{1278}$ & 0,00 & 0.00 & 0.00 & $\infty$ & $\infty 000$ & 000 & $\begin{array}{l}3,587 \pm 0 . \\
02\end{array}$ & $\infty$ & 0.00 & $\infty \infty$ & 0.00 & 000 & ${ }_{000}$ & 0.00 & $\ldots$ & 0.00 & 0.00 & 0.00 & $\infty$ & 0.00 & 0.00 & $\infty$ & 0.00 & 0.00 & 0.00 & ${ }_{000}$ & $\infty$ & $\infty$ & 0.00 & $\infty$ & $\infty$ & 0.00 & 0.00 & 0.00 \\
\hline 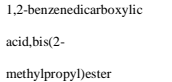 & ${ }_{37008}^{8708}$ & $\infty$ & ${ }_{000}^{000}$ & 0,0 & on & ${ }_{1453}$ & ono & oor & 0,00 & ${ }_{0.00}$ & $0 \infty$ & 000 & 0,0 & oro & ${ }_{0.00}$ & 000 & ${ }_{0.00}^{0}$ & ${ }_{000}$ & 000 & $+\infty$ & ${ }_{0.00}$ & 000 & ${ }_{0 \times 0}$ & 0000 & 0000 & 0.00 & ${ }_{000}$ & ${ }_{\infty \infty}^{\infty}$ & $+\infty$ & 000 & ${ }_{0 \times 0}$ & ${ }_{000}$ & 0000 & 000 & on \\
\hline  & 37.65 & 0.00 & 0.00 & 0.00 & $\infty$ & ${ }_{0000}$ & 0.00 & ${ }_{0000}$ & 0,00 & 0.00 & $\infty, \infty$ & 0.00 & 0,0 & ${ }_{0000}$ & 0.00 & 000 & 0.00 & ${ }_{000}$ & 0.00 & ${ }_{000}$ & ${ }_{0.00}$ & 0.00 & ${ }_{0,0}$ & 0.000 & 0.000 & 0.000 & ${ }_{0000}$ & $\infty$ & $\begin{array}{l}594 \pm \pm 00 \\
4\end{array}$ & 0.000 & ${ }_{000}$ & ${ }_{000}$ & 0.00 & 0.000 & 0.00 \\
\hline 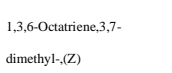 & ${ }_{47.595}$ & 000 & 0.00 & 0.00 & $\infty$ & 0.00 & 30.06 & 0.00 & 0,0 & 0.00 & $\infty, \infty$ & 0.00 & ${ }_{0 \infty}$ & 0.00 & 0.00 & $\begin{array}{l}10235 \pm 0 . \\
06\end{array}$ & 0.00 & +000 & 0.000 & $+\infty 00$ & 0.000 & 0.00 & ${ }_{0,00}$ & 0.000 & 0.000 & 0.000 & ${ }_{000}$ & $\infty$ & $+\infty$ & 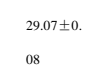 & ${ }_{000}$ & ${ }_{000}$ & 0.00 & 0.000 & 0.00 \\
\hline 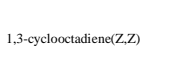 & 2992 & ${ }_{0 \infty}^{\infty}$ & 0.00 & 0.00 & ${ }_{0 \infty}^{\infty}$ & $\begin{array}{l}30360 \pm 0 . \\
04\end{array}$ & 0.00 & 000 & 000 & 0.00 & 0,0 & 0.00 & 0,000 & ${ }_{000}$ & 0.00 & $\infty 000$ & 0.00 & ${ }_{\infty}^{\infty} 00$ & 0.000 & ${ }_{0 \infty 0}$ & 0.000 & 0.00 & ${ }_{0 \times 0}$ & 0.000 & 0.00 & 0.00 & 000 & $\infty$ & ${ }_{\infty}^{\infty}$ & 0.00 & $\begin{array}{l}2001 \pm 000 \\
3\end{array}$ & ${ }_{000}$ & 0.00 & 0.00 & 0.00 \\
\hline 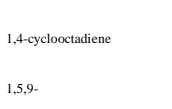 & ${ }_{44417}$ & $\infty$ & 0.00 & 0.00 & 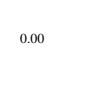 & $\begin{array}{l}28: 4 \pm 0 . \\
06\end{array}$ & ${ }_{11224 \pm 0 .}$ & ${ }_{000}^{\infty}$ & 0,0 & 0.00 & 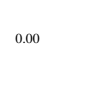 & 0.00 & 0,0 & ${ }_{000}$ & 0.00 & 0.00 & $\begin{array}{l}128 s \pm 00 \\
{ }^{25}\end{array}$ & ${ }_{6.52 \pm 0.5}$ & 0.000 & $+\infty 00$ & 0.000 & 0.00 & ${ }_{000}$ & 0.000 & 0.00 & 0.000 & ${ }_{0000}^{\infty}$ & $\infty$ & ${ }_{000}^{\infty}$ & 0.00 & $\begin{array}{l}31.15 \pm 000 \\
8\end{array}$ & $\begin{array}{l}188 s \pm 0 . \\
03 \\
03\end{array}$ & 0.00 & 0.000 & 0.00 \\
\hline 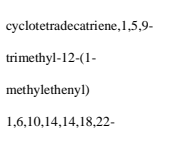 & 30.191 & $\infty \infty$ & 0,0 & 0.00 & $\infty$ & ${ }_{000}$ & $+\infty 00$ & 000 & 000 & 0.00 & 0,0 & 0.00 & $\infty_{0,0}$ & ${ }_{000}$ & 0.00 & 000 & 0.00 & +000 & 0000 & $+\infty$ & 0000 & 0.00 & $\infty$ & 0.000 & 0.00 & 000 & ${ }_{000}$ & ${ }_{\infty \infty}^{\infty}$ & $+\infty$ & 0.00 & $\infty$ & ${ }_{000}$ & 0.000 & 000 & 0.00 \\
\hline 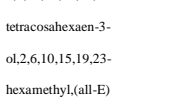 & 47,601 & $\infty$ & 000 & 0.00 & $\infty$ & ${ }_{000}$ & $\infty$ & 000 & 0,0 & 000 & $0 \infty$ & 0.00 & 0,0 & $\infty$ & ono & $\infty$ & ${ }_{0.00}$ & ${ }_{000}^{+\infty}$ & 0.000 & $\infty$ & 0.00 & 0.00 & $\infty$ & 000 & ${ }_{\infty \infty \infty}^{\infty}$ & 0.000 & $\infty \infty$ & $\infty$ & $\infty$ & 0.00 & ${ }_{000}$ & ${ }_{0 \infty 0}$ & 0.00 & 0.00 & 42:5950008 \\
\hline 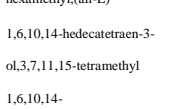 & ${ }_{35587}$ & $\infty$ & 000 & 0.00 & $\infty \infty$ & ${ }_{1054}^{1054 \pm 0}$ & $\infty \infty \infty$ & ono & 000 & 0000 & $0, \infty$ & 000 & 0,00 & $r^{3157 \pm 00}$ & ${ }_{4}^{4 s s 2 x+00}$ & $\begin{array}{l}\text { sassat. } \\
\text { onta. }\end{array}$ & 0.00 & 000 & $\infty$ & sisstao & ${ }_{0000}$ & 0,0 & $\begin{array}{l}18 s 80 \pm 0.0 \\
{ }_{02}\end{array}$ & noro & ${ }^{73,30 \pm 000}$ & no, & 6773500 & $\infty$ & ${ }^{202350 .}$ & $\begin{array}{l}16321 \pm 0 \\
{ }_{208}\end{array}$ & $\infty$ & 000 & 0.00 & 0,000 & 0.00 \\
\hline 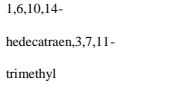 & ${ }_{48357}^{437}$ & $0 \infty$ & ${ }_{000}$ & 0.00 & $\infty \infty$ & $\begin{array}{l}80,7 \\
07\end{array}$ & 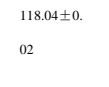 & ${ }_{000}$ & 000 & 0,0 & 000 & $\infty 000$ & $\infty$ & ${ }_{000}$ & ono & 0000 & 000 & ono & $00 \infty$ & $+\infty$ & ${ }_{000}$ & $\begin{array}{l}3805 \pm 000 \\
3\end{array}$ & ${ }_{000}$ & nor & 000 & 0.00 & 000 & $\infty$ & $\infty \infty$ & 0.00 & 000 & 000 & 0,00 & 000 & 0.00 \\
\hline 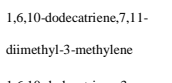 & ${ }_{43267}^{4267}$ & 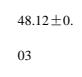 & $\begin{array}{l}100655 \pm \\
004\end{array}$ & $\begin{array}{l}311: 22 \pm 0 . \\
04\end{array}$ & 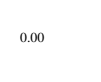 & ono & 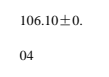 & 0.00 & 0.000 & 000 & $\begin{array}{l}55737 \pm 00 \\
2\end{array}$ & 0.00 & $\begin{array}{l}100.10 \pm 0.0 . \\
02.0\end{array}$ & ouo & 0.000 & 000 & $\begin{array}{l}5206 \pm 000 \\
8\end{array}$ & 000 & $\begin{array}{l}37,5 \pm 0.0 . \\
03\end{array}$ & $\begin{array}{l}9 .+13 \pm 00 \\
2\end{array}$ & 0.000 & 0.00 & ${ }_{0 \times 0}$ & $\begin{array}{l}\text { sos } 08.0 . \\
05\end{array}$ & 0.00 & 0.000 & oro & $\begin{array}{l}2982 \pm 00 \\
4\end{array}$ & $\begin{array}{l}13: 560.0 . \\
05\end{array}$ & 0.00 & ono &  & 0000 & 0000 & $\begin{array}{l}203236 \pm 00 \\
5\end{array}$ \\
\hline 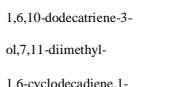 & ${ }_{4,355}^{435}$ & $\infty 00$ & ono & als:sta. & $\begin{array}{l}{ }^{46,457 \pm 00} \\
3\end{array}$ & $0 \infty 0$ & $199+0005$ & 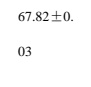 & $e^{m 77 \pm 000}$ & 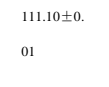 & $\infty 000$ & 000 & $\infty$ & $0 \infty$ & ono & 0000 & ono & 000 & 000 & 000 & 000 & 0000 & ${ }_{0000}$ & $\begin{array}{l}21.7 .7 \pm 0 . \\
05\end{array}$ & 000 & 0.00 & oon & $\infty$ & 0000 & 000 & ${ }_{0000}$ & 000 & $\infty 00$ & 0.00 & 0.00 \\
\hline 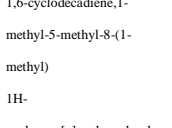 & ${ }_{38356}^{336}$ & 0,0 & 0.00 & 0.00 & ${ }_{\infty \infty \infty}$ & ${ }_{000}$ & +on & 000 & 000 & 0.00 & 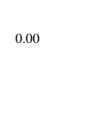 & 0.00 & 0,0 & 000 & 0.00 & 0.00 & 0.00 & ${ }_{000}^{\infty}$ & 0.000 & $+\infty$ & 0.00 & 0.00 & ${ }_{0 \times 0}$ & 0.000 & 0.00 & 0.00 & ${ }_{000}$ & $\infty$ & $+\infty 00$ & 0.000 & ${ }_{0000}$ & ${ }_{\infty \infty 00}$ & 0.00 & ${ }_{000}$ & 0.00 \\
\hline  & 29895 & 0,0 & 0.00 & 0,0 & on & ${ }_{0 \infty \infty}$ & ono & oon & 0,00 & 0.00 & $0, \infty$ & $\infty 000$ & 0,00 & oor & 0.00 & 000 & 0.00 & ${ }_{000}^{\infty}$ & 0.000 & $+\infty$ & 0.00 & 0,00 & ${ }_{000}$ & 0.000 & 0.00 & 0.00 & 000 & ${ }_{\infty \infty}^{\infty}$ & ${ }_{\infty}^{\infty}$ & 0.00 & ${ }_{0000}$ & ${ }_{000}$ & 0.00 & 0.000 & 0.00 \\
\hline 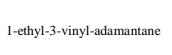 & 21.656 & 0,00 & 0.00 & 0,00 & $0 \infty$ & 0,00 & 000 & 000 & $783 \pm 0.0$ & 0.000 & $0, \infty$ & 0.00 & 0,000 & ${ }_{000}$ & 0.00 & 000 & 0.00 & 000 & 0.000 & $\infty+00$ & 0.00 & 0.00 & 000 & 0.00 & 0,000 & 0.00 & 000 & $\infty$ & 0.00 & 0.00 & 000 & 000 & 0.000 & 0.00 & 0.00 \\
\hline
\end{tabular}




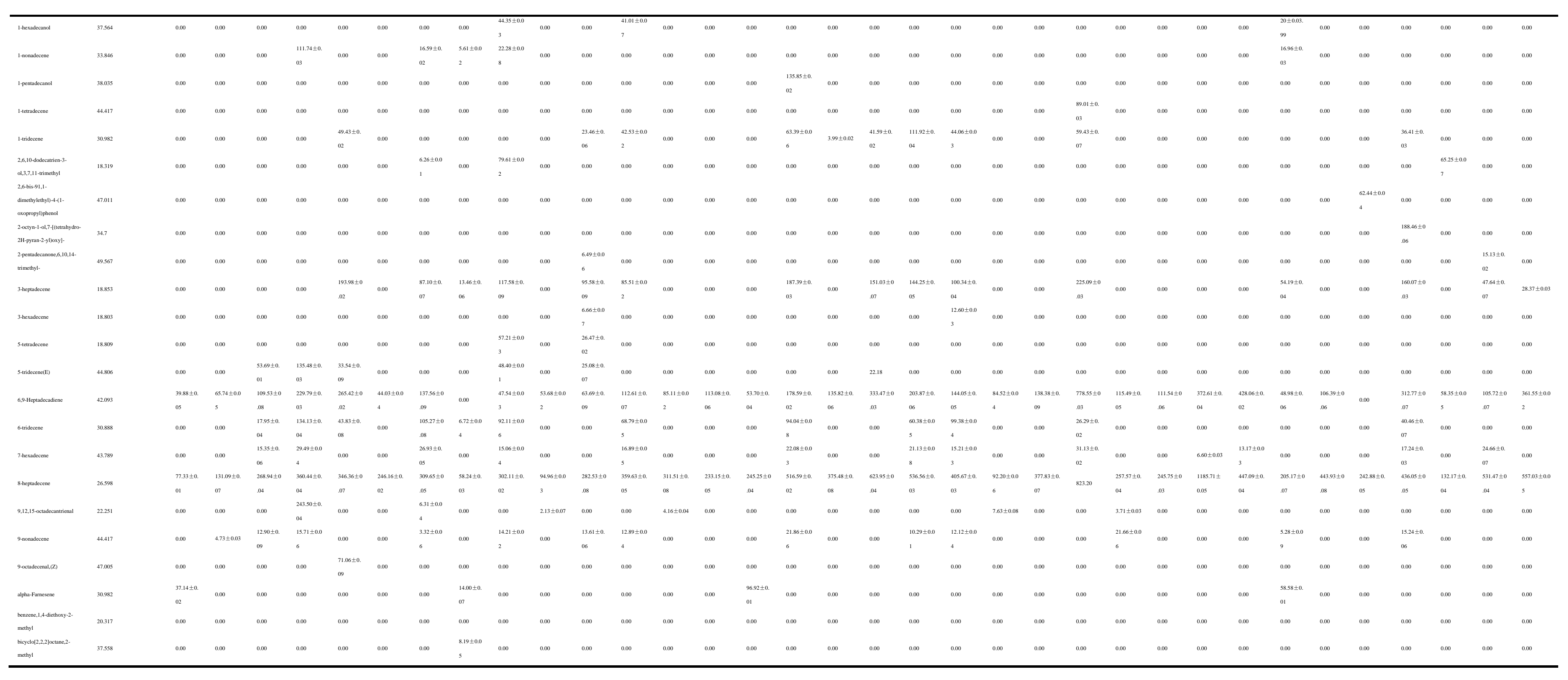









\begin{tabular}{|c|c|c|c|c|c|c|c|c|c|c|c|c|c|c|c|c|c|c|c|c|c|c|c|c|c|c|c|c|c|c|c|c|c|c|c|}
\hline 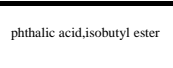 & 43739 & 0,000 & 000 & 8000 & 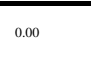 & 0.000 & 0,000 & 0,000 & 0000 & $5 s 4 \pm 0.03$ & 0,0 & $\begin{array}{l}3.1887 \\
04 \\
04\end{array}$ & 0,000 & $4900 \pm 001$ & 0.000 & 0,000 & 0.000 & 0,000 & 0.000 & 0,000 & 200 & $\frac{2121}{2}$ & 0,000 & 0000 & 0,000 & 000 & 0,000 & 000 & 000 & 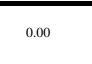 & 0000 & 0.00 & 0000 & 000 & 0000 \\
\hline 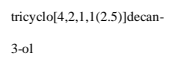 & 37772 & 0.000 & $\infty_{0 \infty}$ & 0,000 & $\begin{array}{l}180.04 \pm 0.0 \\
6\end{array}$ & $\begin{array}{l}18.17 \pm 0 . \\
06\end{array}$ & 000 & ${ }_{0 \infty}$ & 0,00 & oro & $0 \infty$ & $\infty 000$ & ${ }_{000}$ & ${ }_{000}$ & 0.00 & 000 & 0.00 & 000 & 0.00 & 000 & 000 & 0.00 & ${ }_{000}^{\infty}$ & 000 & 000 & ${ }_{0 \infty 0}$ & 000 & 0,00 & ${ }_{0 \infty 0}^{\infty}$ & 0.00 & $\infty$ & 0.00 & ${ }_{000}^{\infty}$ & ${ }_{0 \infty 0}$ & ${ }_{0000}$ \\
\hline urarascenala & ${ }_{42677}$ & 0.000 & $\infty . \infty$ & ${ }^{2603 \pm 0.0}$ & $9200 \pm 00$ & $\begin{array}{l}6501 \pm 0 . \\
04\end{array}$ & 000 & $\begin{array}{l}58.150 . \\
05\end{array}$ & $\begin{array}{l}1422 \pm \pm 0 . \\
03\end{array}$ & ${ }_{14423 \pm 0 .}^{1140 .}$ & $9322 \pm 0.04$ & $\infty 00$ & $\infty \ldots$ & $43,4,4 \pm 00$ & $\begin{array}{l}2.14 \pm \pm 00 \\
3\end{array}$ & 000 & 0.000 & 000 & 0.00 & 0.00 & 0.00 & 0.000 & 0,000 & 000 & 0,000 & $\begin{array}{l}5274 \pm 0 . \\
05\end{array}$ & 0,000 & 0,00 & $\infty 000$ & 0,000 & 0000 & 0.00 & $\begin{array}{l}2821 \pm 00 \\
1\end{array}$ & 000 & 000 \\
\hline uerantacene & 265988 & 0000 & 0,0 & 0.00 & 000 & 0.00 & 000 & $\infty$ & 000 & $\infty$ & $0 \infty$ & $\begin{array}{l}380070.9 . \\
08 \\
08\end{array}$ & 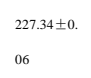 & 000 & $\begin{array}{l}20.53 \pm 000 \\
2^{2}\end{array}$ & 000 & ${ }_{6,35 s \pm 00}$ & 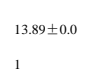 & 11090900 & 16s:ssta. & $9596 \%+00$ & 0.00 & 0,00 & 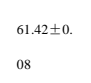 & ${ }_{s: 47 \pm 00}$ & 0.00 & 0000 & 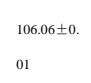 & $\begin{array}{l}1236 \pm 0 . \\
{ }_{18}\end{array}$ & $\infty \infty$ & $\begin{array}{l}186 \cap \pm 000 \\
s\end{array}$ & ${ }_{0.5}^{2 s s \pm 0 .}$ & ${ }_{0000}$ & $\begin{array}{l}1,1,2 \pm 0 . \\
09\end{array}$ & $9192 \pm$ \\
\hline 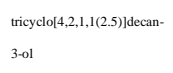 & 378.87 & 0.00 & $0 \infty$ & 0,0 & 000 & 0.00 & 000 & 000 & 000 & 0.00 & $\infty$ & $\infty 000$ & 0,0 & ${ }_{000}$ & 0.00 & 000 & 000 & 000 & 0.00 & 000 & $\infty 000$ & ${ }_{000}$ & ${ }_{0 \times 0}$ & $\begin{array}{l}62009 \pm 0 . \\
01\end{array}$ & 0,00 & 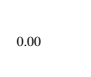 & 000 & $\infty$ & $\infty$ & 000 & 000 & $\infty \infty$ & 0.00 & 000 & ${ }_{000}$ \\
\hline mincecenc & 22251 & 0.00 & ot &  & $\begin{array}{l}6019.9 \pm 0.0 . \\
08\end{array}$ & $\begin{array}{l}125.34 \pm 0 \\
.03\end{array}$ & $280.5 \pm 0.0$ & $\begin{array}{l}89,13 \pm 0 . \\
07\end{array}$ & $\begin{array}{l}41,93 \pm 0 . \\
01\end{array}$ & $\begin{array}{l}1535 s+a . \\
{ }_{05}\end{array}$ & $\begin{array}{l}1029 \pm 0.0 \\
4\end{array}$ & $\begin{array}{l}130.2 \pm 0 \\
.09\end{array}$ & 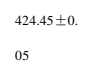 & ${ }_{0000}$ & $\begin{array}{l}13,35 \pm 00 \\
1\end{array}$ & 0000 & 59670 & $9 r 7 \pm 001$ & $24795 \pm 0$ & $\begin{array}{l}18807+ \pm .0 . \\
03\end{array}$ & 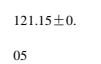 & 0.00 & ${ }_{0 \times 0}$ & $\begin{array}{l}206321 \pm 0 \\
02 \\
02\end{array}$ & $\begin{array}{l}76293 \pm 00 \\
2\end{array}$ & 0,00 & 000 & $\begin{array}{l}15211 \pm 0 . \\
002\end{array}$ & $\begin{array}{l}102985 \pm 0 \\
07\end{array}$ & 000 & $1974 \pm 000$ & $\begin{array}{l}5 s, 43 \pm 0 . \\
0.5\end{array}$ & $\begin{array}{l}1,1,800 \pm 000 \\
0\end{array}$ & $\begin{array}{l}20.560 \pm 0 . \\
07\end{array}$ & $5697 \pm 006$ \\
\hline uncreane & 14721 & 0000 & $\infty$ & 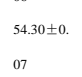 & $\begin{array}{l}52.19 \pm 00 \\
8\end{array}$ & $\begin{array}{l}17988 \pm 0 . \\
06\end{array}$ & 000 & $\begin{array}{l}7927 \pm 0 . \\
02\end{array}$ & $\begin{array}{l}92595 \pm .0 . \\
{ }_{08}\end{array}$ & $\begin{array}{l}198,19 \pm 0.0 \\
07 \\
07\end{array}$ & $\begin{array}{l}19000 \pm 000 \\
2\end{array}$ & ${ }_{250030 \pm 0}^{230}$ &  & 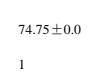 & 000 & 22160.0. & m7701 & $3386 \pm 00$ & 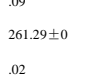 & 2135 sous & 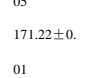 & 0.00 & ${ }_{0,00}$ & 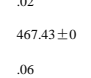 & $\begin{array}{l}20577 \pm 0 . \\
07 \\
07\end{array}$ & $\infty$ & $\begin{array}{l}1281 \pm 00 \\
3 \\
3\end{array}$ & 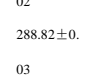 & 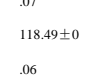 & $\begin{array}{l}9607 \pm 0 . \\
01\end{array}$ & $2421 \pm 00$ & 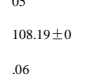 & $3^{32620 \pm 000}$ & ssi:2.2. & $19983 \pm 00$ \\
\hline 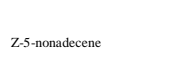 & ${ }_{44.417}$ & 0.00 & ${ }_{000}$ & $\begin{array}{l}18>70 \pm 0 . \\
0.4 \\
0.4\end{array}$ & $\begin{array}{l}13.19 \pm 00 \\
6\end{array}$ & $\begin{array}{l}1483 \pm 0 . \\
03 \pm 0 .\end{array}$ & 0.00 & $\begin{array}{l}13: 0.9 \pm 0.0 \\
0 .\end{array}$ & $\begin{array}{l}12322 \pm 0 . \\
06\end{array}$ &  & $0_{0 \infty}$ & $\begin{array}{l}21.61 \pm 0 . \\
02\end{array}$ & $\begin{array}{l}11922 \pm 0.0 \\
3\end{array}$ & $\begin{array}{l}1098 \pm 000 \\
1\end{array}$ & 0.00 & 000 & ${ }_{1702}$ & $\begin{array}{l}23,59 \pm \pm 0.0 \\
4\end{array}$ & $\begin{array}{l}16,69 \pm 0 . \\
02 \\
02\end{array}$ & $\begin{array}{l}31 s \pm \pm 00 \\
5\end{array}$ & $\begin{array}{l}1227 \pm 00 \\
4\end{array}$ & 0.00 & 0000 & $\begin{array}{l}4198 \pm \pm .0 . \\
{ }_{08}\end{array}$ & 000 & $\begin{array}{l}8.55 \pm 0.0 \\
2\end{array}$ & $\begin{array}{l}11.03 \pm 0.0 \\
3\end{array}$ & $\begin{array}{l}2438 \pm 000 \\
4\end{array}$ & $\begin{array}{l}18003 \pm 0 . \\
05\end{array}$ & 0.000 & $\begin{array}{l}1032 \pm 00 \\
2 \\
2\end{array}$ & & ${ }_{0,00}$ & ${ }_{0 \infty}$ & \\
\hline
\end{tabular}

Supplemental Table 2. Types and concentrations (ng/g) of volatile compounds in tropical water lily flowers. Cultivars are coded as in Table 1.

\begin{tabular}{|c|c|c|c|c|c|c|c|c|c|c|c|c|c|c|c|c|c|c|c|c|c|c|c|}
\hline Compounds & (min) & ${ }_{\mathrm{R} 1}$ & $\mathrm{R} 2$ & $\mathrm{R} 3$ & ${ }_{\mathrm{R} 4}$ & R5 & R6 & R7 & R8 & R9 & R10 & RII & R12 & R13 & R14 & R15 & $\mathrm{R} 16$ & R17 & R18 & R19 & R20 & R21 & R22 \\
\hline (E,E,E)-3,7,11,15-teramenehylhexadeca-1,3,6,10,14-pentaene & 45.001 & 0.00 & 0.00 & 0.00 & 0.00 & 0.00 & 0.00 & 0.00 & 0.00 & 0.00 & 0.00 & 0.00 & 0.00 & $\begin{array}{l}0.00 \\
76.63 \pm 0.0\end{array}$ & 0.00 & 0.00 & 0.00 & 0.00 & 0.00 & 0.00 & $\begin{array}{l}31.57 \pm 0.0 \\
5\end{array}$ & 0.00 & 0.00 \\
\hline 1,11-dodecadien & 49.046 & 0.00 & 0.00 & 0.00 & 0.00 & 0.00 & 0.00 & 0.00 & 0.00 & 0.00 & 0.00 & 0.00 & 0.00 & 9 & 0.00 & 0.00 & 0.00 & 0.00 & 0.00 & $5.84 \pm 0.05$ & 0.00 & 0.00 & 0.00 \\
\hline  & 47.955 & $5.27 \pm 0.01$ & 0.00 & 0.00 & $13.82 \pm 0.08$ & $11.56 \pm 0.04$ & 0.00 & $\begin{array}{l}0.00 \\
2169.69 \pm 0 .\end{array}$ & $9.69 \pm 0.02$ & 0.00 & 0.00 & 0.00 & 0.00 & 0.00 & 0.00 & 0.00 & 0.00 & 0.00 & 0.00 & 0.00 & 0.00 & 0.00 & 0.00 \\
\hline 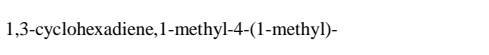 & 47.995 & 0.00 & 0.00 & 0.00 & 0.00 & 0.00 & 0.00 & 02 & 0.00 & $13.10 \pm 0.02$ & 0.00 & 0.00 & 0.00 & 0.00 & 0.00 & 0.00 & 0.00 & 0.00 & 0.00 & 0.00 & 0.00 & 0.00 & 0.00 \\
\hline 1.3-cyclohexadiene, 5 -(1,5.-dimethyl) & 47.589 & $88.67 \pm 0.02$ & $20.07 \pm 0.08$ & $69.89 \pm 0.09$ & $43.84 \pm 0.06$ & $38.76 \pm 0.03$ & 0.00 & 0.00 & $39.88 \pm 0.01$ & $9.9 \pm \pm 0.01$ & $\begin{array}{l}0.00 \\
189.14 \pm 0.0\end{array}$ & 0.00 & 0.00 & 0.00 & 0.00 & 0.00 & 0.00 & 0.00 & 0.00 & 0.00 & 0.00 & 0.00 & 0.00 \\
\hline 1,3,6,10-dodeceateranen,3,7,11-rimenthy-(Z,E) & 47.955 & 0.00 & 0.00 & 0.00 & 0.00 & 0.00 & 0.00 & 0.00 & 0.00 & 0.00 & 4 & $\begin{array}{l}0.00 \\
3138.89 \pm 0 .\end{array}$ & 0.00 & 0.00 & 0.00 & 0.00 & 0.00 & 0.00 & 0.00 & $21.51 \pm 0.04$ & 0.00 & $19.73 \pm 0.04$ & 0.00 \\
\hline 1,3-cyclohexadiene,5-5(1,5-dimethyl-4hexenyl)-2-methyl & 28.802 & 0.00 & 0.00 & 0.00 & 0.00 & 0.00 & 0.00 & 0.00 & 0.00 & 0.00 & $63.35 \pm 0.04$ & 05 & 0.00 & 0.00 & $97.38 \pm 0.04$ & $22.86 \pm 0.05$ & $51.59 \pm 0.04$ & $\begin{array}{l}0.00 \\
254.50 \pm 0.0\end{array}$ & $18.58 \pm 0.05$ & 0.00 & 0.00 & $78.30 \pm 0.05$ & 0.00 \\
\hline 1.3-cyclooctadiener(Z,Z) & 29.92 & 0.00 & 0.00 & 0.00 & 0.00 & 0.00 & 0.00 & 0.00 & 0.00 & 0.00 & 0.00 & 0.00 & 0.00 & 0.00 & 0.00 & 0.00 & 0.00 & 5 & 0.00 & 0.00 & 0.00 & 0.00 & 0.00 \\
\hline
\end{tabular}


1.4-cyclooctadiene

1.5.5.trimethy-6-mentylyenc-yclohexene

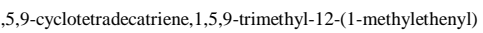

1.59.-cyclododecarariene, $1,5,9$-rimethy

1,6,10,14-1-edecatran, $3,7,11$-rimethy

1,6,10-dodecatriene,71-1-dimethy 1--methylene

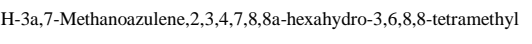

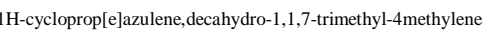

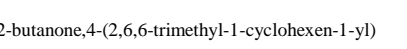

1--nonadecene

1-tridecen

2-hepradecanone

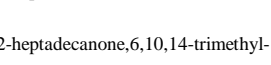

2-octene,(E)

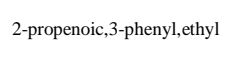

3,4-octadiene, 7 -methyl-

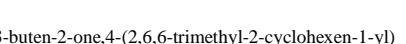

5-terradecene

6.9-Heppadecadiene $\begin{array}{rlrrr}187.56 \pm 0.0 & 292.80 \pm 0.0 & 85.99 \pm 0.0 & 187.63 \pm 0.0 & 14.7 .5 \pm \pm 0.0\end{array}$



$\begin{array}{llllllll}30.191 & 0.00 & 0.00 & 0.00 & 0.00 & 0.00 & 0.00 & 0.00\end{array}$



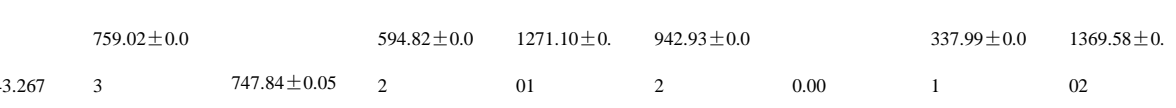

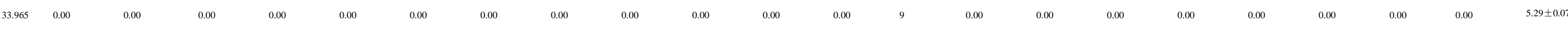

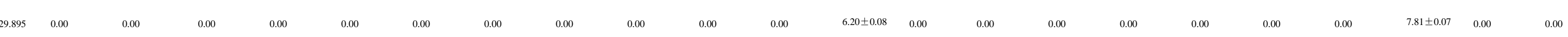

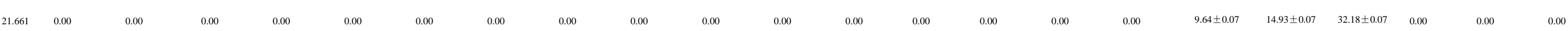

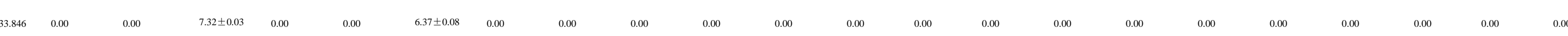

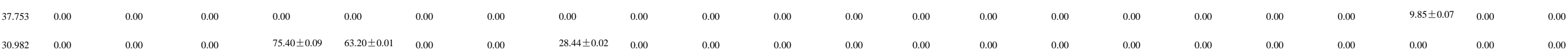

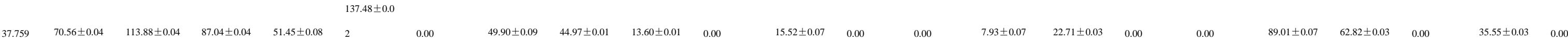

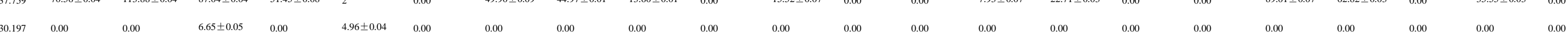

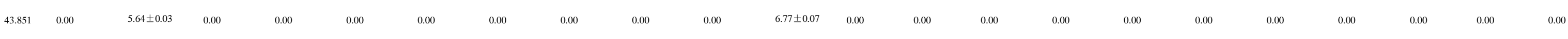

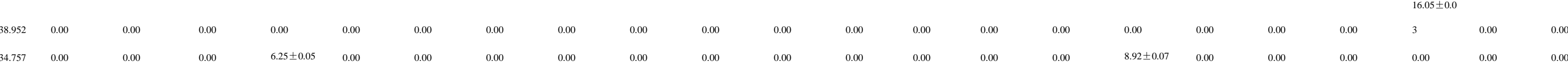

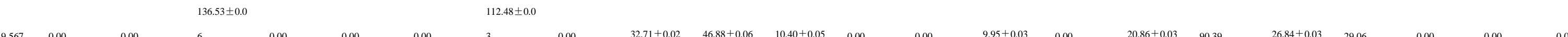

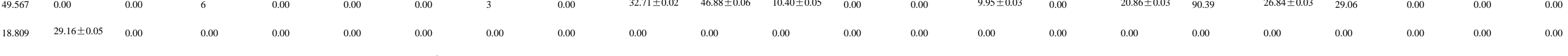

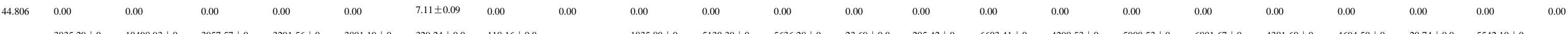

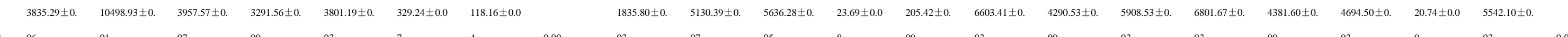
$\begin{array}{lllllllllllllllllllllll}42.93 & 06 & 01 & 07 & 09 & 03 & 7 & 4 & 0.00 & 03 & 07 & 05 & 8 & 09 & 03 & 09 & 03 & 03 & 09 & 03 & 9 & 03 & 0.03\end{array}$

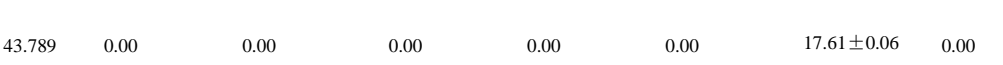




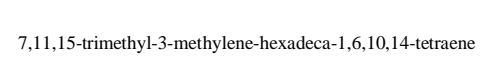
8.-heparaceene

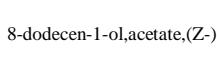

9,12,15-coctadecantrienal

9,12-0catadeadienonic acid, methy sester

9,17-cradecadienal,(Z-)

9.-icosyne

nectic acid.phenymmethy ester

ulpha-Farnesene

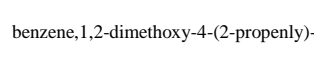

benzene.1,1,3,-5rtinethoxy

benzene, 1,4-diethoxy-2-methyl

benzoic acid, ehyly ester

benzyy benroate

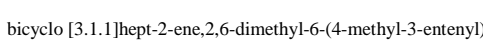



bityclo[3.3.11 Inomane

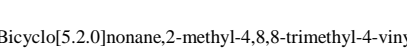
bieyclo[5.3.0|decane

cis,cis, cis-7,7,1,13.1-hexadecartienal

is -7-dodecen-1-yl acectate

Cyclobutanecarboxylic





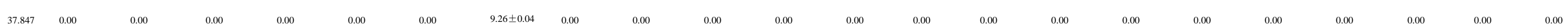

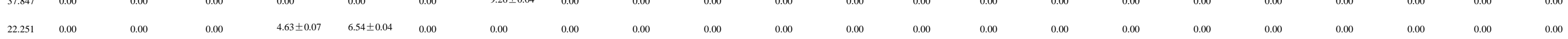

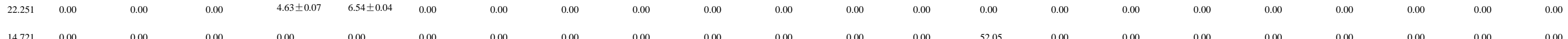

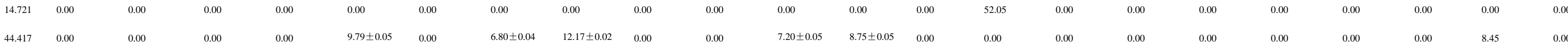

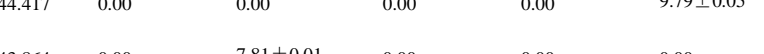

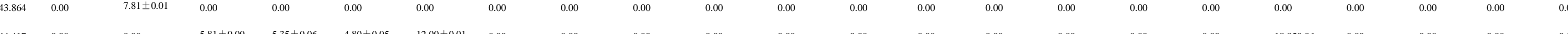

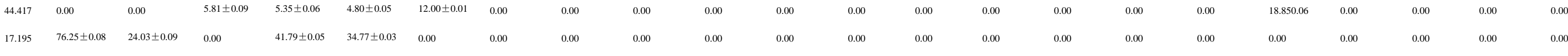

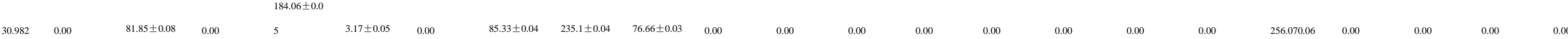

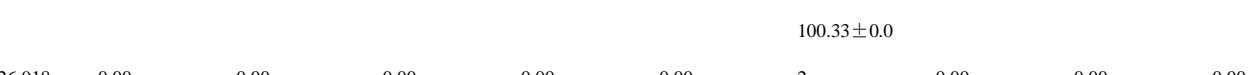

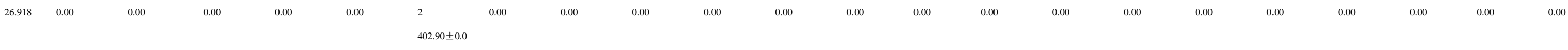

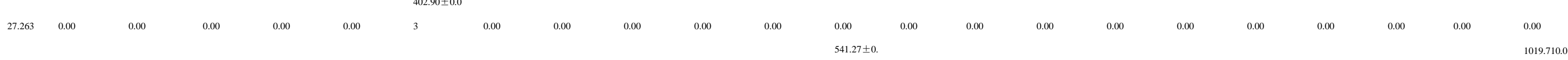

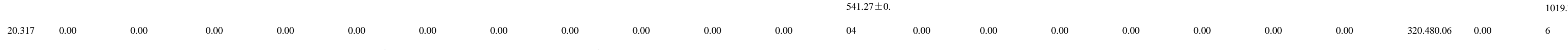



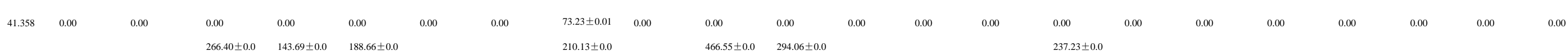

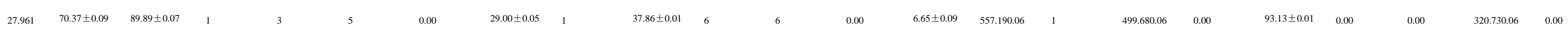

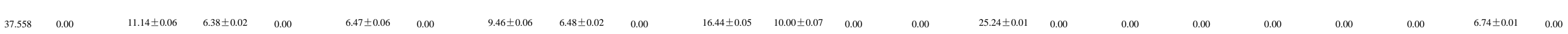

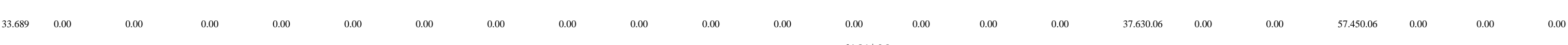

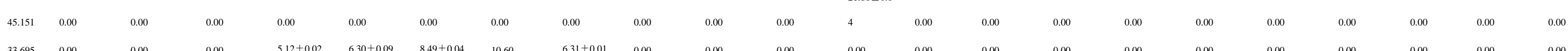

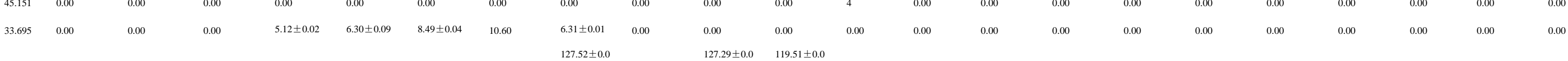

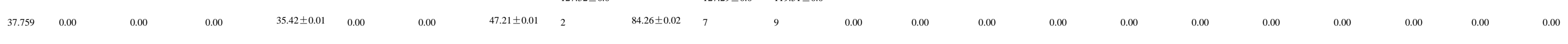

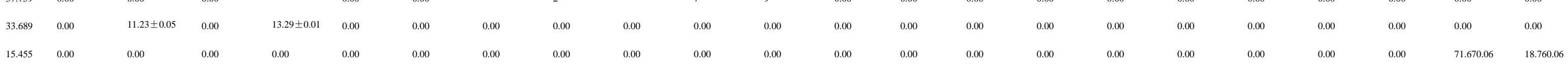




\begin{tabular}{|c|c|c|c|c|c|c|c|c|c|c|c|c|c|c|c|c|c|c|c|c|c|c|c|}
\hline Cyclobutanecarboxylic acid,octy lester & 15.487 & $51.65 \pm 0.05$ & 0.00 & 0.00 & $9.44 \pm 0.02$ & $34.44 \pm 0.08$ & 0.00 & 0.00 & 0.00 & 0.00 & 0.00 & 0.00 & 0.00 & 0.00 & 0.00 & 0.00 & 0.00 & 0.00 & 0.00 & 0.00 & 0.00 & 0.00 & $\begin{array}{l}0.00 \\
13.76 \pm 0.0\end{array}$ \\
\hline cyclodecene & 37.558 & 0.00 & $12.55 \pm 0.04$ & $7.57 \pm 0.03$ & $10.67 \pm 0.04$ & $6.89 \pm 0.07$ & $33.06 \pm 0.03$ & $7.42 \pm 0.02$ & 0.00 & 0.00 & $32.32 \pm 0.05$ & $17.37 \pm 0.07$ & 0.00 & 0.00 & 0.00 & $16.84 \pm 0.01$ & 0.00 & 0.00 & 49.84 & 0.00 & 59.12 & 0.00 & 8 \\
\hline 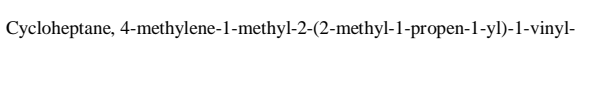 & 45.529 & 0.00 & 0.00 & 0.00 & 0.00 & 0.00 & $7.49 \pm 0.01$ & 0.00 & 0.00 & 0.00 & 0.00 & 0.00 & 0.00 & $\begin{array}{l}0.00 \\
22.37 \pm 0.0\end{array}$ & 0.00 & $\begin{array}{l}0.00 \\
1022.76 \pm 0.0\end{array}$ & 0.00 & 0.00 & 0.00 & 0.00 & 0.00 & 0.00 & 0.00 \\
\hline cyclohexene, I- I-mehtyl.4.(1-mentylehylidene) & 14.193 & 0.00 & 0.00 & 0.00 & 0.00 & 0.00 & 0.00 & 0.00 & 0.00 & 0.00 & 0.00 & 0.00 & 0.00 & 9 & 0.00 & 6 & 0.00 & 0.00 & 0.00 & 0.00 & 0.00 & 0.00 & 0.00 \\
\hline 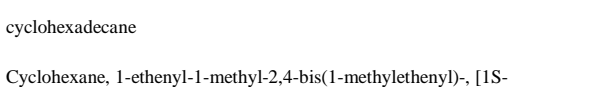 & 47.293 & 0.00 & 0.00 & $16.15 \pm 0.04$ & 0.00 & 0.00 & 0.00 & 0.00 & 0.00 & $12.04 \pm 0.02$ & 0.00 & 0.00 & $\begin{array}{l}0.00 \\
266.48 \pm 0 .\end{array}$ & 0.00 & 0.00 & 0.00 & 0.00 & 0.00 & 0.00 & 0.00 & $\begin{array}{l}0.00 \\
433.39+0.0\end{array}$ & 0.00 & 0.00 \\
\hline 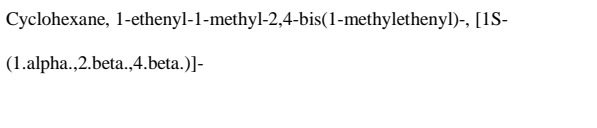 & 45.949 & $\begin{array}{l}0.00 \\
361.07 \pm 0.0\end{array}$ & 0.00 & $\begin{array}{l}0.00 \\
394.4 \pm \pm 0.0\end{array}$ & $\begin{array}{l}0.00 \\
205.50 \pm 0.0\end{array}$ & $\begin{array}{l}0.00 \\
247.7 \pm 0.0\end{array}$ & $66.70 \pm 0.03$ & 0.00 & $\begin{array}{l}0.00 \\
266.62 \pm 0.0\end{array}$ & 0.00 & $\begin{array}{l}0.00 \\
1203.84 \pm 0 .\end{array}$ & $\begin{array}{l}0.00 \\
376.11 \pm 0.0\end{array}$ & 08 & $\begin{array}{l}0.00 \\
38.15 \pm 0.0\end{array}$ & 0.00 & 0.00 & 0.00 & 0.00 & $\begin{array}{l}0.00 \\
225.89 \pm 0.0\end{array}$ & 0.00 & 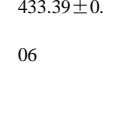 & $\begin{array}{l}0.00 \\
711.47 \pm 0.0\end{array}$ & \\
\hline 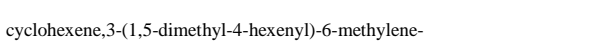 & 31.691 & 7 & $241.12 \pm 0.03$ & 5 & 3 & 9 & 0.00 & $41.69 \pm 0.01$ & 2 & $31.24 \pm 0.02$ & 06 & 6 & 0.00 & 4 & $27.56 \pm 0.06$ & 316.52 & $21.78 \pm 0.06$ & $7.40 \pm 0.01$ & 1 & 0.00 & 0.00 & 6 & $7.53 \pm 0.08$ \\
\hline cyclopentadceane & 30.197 & 0.00 & $58.24 \pm 0.02$ & 0.00 & $31.37 \pm 0.03$ & $13.92 \pm 0.08$ & $28.64 \pm 0.06$ & 0.00 & 0.00 & $7.63 \pm 0.01$ & 0.00 & 0.00 & 0.00 & 0.00 & 0.00 & 0.00 & $\begin{array}{l}0.00 \\
144.67 \pm 0.0\end{array}$ & 0.00 & 0.00 & 0.00 & 0.00 & $83.00 \pm 0.01$ & 0.00 \\
\hline cycloteradecane & 30.039 & 0.00 & 0.00 & 0.00 & 0.00 & 0.00 & 0.00 & 0.00 & 0.00 & 0.00 & 0.00 & 0.00 & 0.00 & 0.00 & 0.00 & 0.00 & 8 & 0.00 & 0.00 & 0.00 & 0.00 & 0.00 & 0.00 \\
\hline E-7-dodecen-1-ol acetate & 33.557 & 0.00 & 0.00 & 0.00 & 0.00 & 0.00 & $6.18 \pm 0.02$ & 0.00 & 0.00 & 0.00 & 0.00 & 0.00 & 0.00 & 0.00 & 0.00 & 0.00 & 0.00 & 0.00 & 0.00 & 0.00 & 0.00 & 0.00 & 0.00 \\
\hline 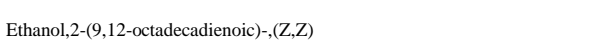 & 33.689 & 0.00 & 0.00 & 0.00 & 0.00 & 0.00 & 0.00 & 0.00 & 0.00 & 0.00 & 0.00 & 0.00 & 0.00 & 0.00 & 0.00 & 0.00 & 0.00 & $33.00 \pm 0.01$ & $4.69 \pm 0.01$ & $15.94 \pm 0.01$ & 0.00 & $6.78 \pm 0.01$ & 0.00 \\
\hline eicosane & 47.011 & 0.00 & 0.00 & 0.00 & 0.00 & 0.00 & $7.13 \pm 0.03$ & 0.00 & 0.00 & 0.00 & 0.00 & 0.00 & 0.00 & $\begin{array}{l}0.00 \\
62.00 \pm 0.0\end{array}$ & 0.00 & 0.00 & 0.00 & 0.00 & 0.00 & 0.00 & 0.00 & 0.00 & 0.00 \\
\hline heneicosane & 49.567 & $\begin{array}{l}28.24 \pm 0.04 \\
203.36 \pm 0.0\end{array}$ & $40.56 \pm 0.01$ & $\begin{array}{l}30.37 \pm 0.06 \\
403.23 \pm 0.0\end{array}$ & $\begin{array}{r}33.99 \pm 0.05 \\
149.79 \pm 0.0\end{array}$ & $\begin{array}{l}3.6 .85 \pm 0.08 \\
163.96 \pm 0.0\end{array}$ & $92.83 \pm 0.04$ & $\begin{array}{l}17.70 \pm 0.02 \\
125.26 \pm 0.0\end{array}$ & $\begin{array}{l}36.37 \pm 0.03 \\
137.10 \pm 0.0\end{array}$ & $\begin{array}{l}19.34 \pm 0.02 \\
189.60 \pm \pm 0.0\end{array}$ & $\begin{array}{l}17.75 \pm 0.06 \\
252.47 \pm 0.0\end{array}$ & $\begin{array}{l}21.51 \pm 0.06 \\
177.61 \pm 0.0\end{array}$ & $\begin{array}{l}0.00 \\
69.06 \pm 0.0\end{array}$ & $\begin{array}{l}4 \\
24.05 \pm 0.0\end{array}$ & $\begin{array}{l}16.55 \pm 0.08 \\
228.47 \pm 0.0\end{array}$ & $\begin{array}{l}22.99 \\
105.04 \pm 0.0\end{array}$ & $\begin{array}{l}15.41 \pm 0.08 \\
230.41 \pm 0.0\end{array}$ & $\begin{array}{l}13.41 \\
683.42 \pm 0.0\end{array}$ & $\begin{array}{r}24.12 \pm 0.08 \\
202.19 \pm 0.0\end{array}$ & $\begin{array}{l}20.82 \\
20.999 \pm 0.0\end{array}$ & $\begin{array}{l}7.88 \\
171.83 \pm 0 .\end{array}$ & $\begin{array}{l}44.28 \\
211.94 \pm 0.0\end{array}$ & $\begin{array}{l}6.69 \pm 0.08 \\
23.61 \pm 0.0\end{array}$ \\
\hline hepatadcane & 38.952 & 6 & $212.01 \pm 0.09$ & 7 & 6 & 7 & $33.82 \pm 0.05$ & 3 & 3 & 2 & 6 & 5 & $\begin{array}{l}4 \\
17.98 \pm 0.0\end{array}$ & 4 & 2 & ${ }^{2}$ & 2 & 2 & 2 & 2 & $\begin{array}{l}02 \\
40.11 \pm 0.0\end{array}$ & 2 & 8 \\
\hline hexadecane & 34.757 & 0.00 & $62.27 \pm 0.08$ & $10.58 \pm 0.08$ & $10.09 \pm 0.04$ & $11.24 \pm 0.06$ & $9.90 \pm 0.06$ & $13.77 \pm 0.08$ & $9.95 \pm 0.03$ & 0.00 & $17.30 \pm 0.07$ & $10.13 \pm 0.05$ & 8 & $\begin{array}{l}0.00 \\
27.80 \pm 0.0\end{array}$ & 0.00 & 0.00 & $8.45 \pm 0.06$ & $34,43 \pm 0.07$ & 0.00 & $25.25 \pm 0.06$ & 7 & $15.04 \pm 0.06$ & 0.00 \\
\hline kaurene & 48.135 & $\begin{array}{l}0.00 \\
127.14 \pm 0.0\end{array}$ & $5.93 \pm 0.07$ & $\begin{array}{l}8.59 \pm 0.09 \\
131.36 \pm 0.0\end{array}$ & $\begin{array}{l}6.41 \pm 0.08 \\
1116.28 \pm 0.0\end{array}$ & $\begin{array}{l}5.86 \pm 0.05 \\
111.86 \pm 0.0\end{array}$ & $96.50 \pm 0.07$ & $6.74 \pm 0.07$ & $\begin{array}{l}6.71 \pm 0.04 \\
124.79 \pm 0.0\end{array}$ & $7.11 \pm 0.08$ & $6.77 \pm 0.06$ & $5.79 \pm 0.08$ & 0.00 & $\begin{array}{l}4 \\
49.98 \pm 0.0\end{array}$ & $8.42 \pm 0.02$ & $5.72 \pm 0.07$ & $9.62 \pm 0.07$ & $16.57 \pm 0.07$ & $15.25 \pm 0.07$ & $37.98 \pm 0.07$ & $\begin{array}{l}0.00 \\
30.16 \pm 0.0\end{array}$ & $\begin{array}{l}5.90 \pm 0.07 \\
155.76 \pm 0.0\end{array}$ & 0.00 \\
\hline nonadecane & 44.806 & 4 & $142.34 \pm 0.05$ & 1 & ${ }^{3}$ & ${ }^{5}$ & $39.80 \pm 0.08$ & $38.18 \pm 0.04$ & 5 & $62.07 \pm 0.02$ & $59.05 \pm 0.05$ & $68.02 \pm 0.09$ & $6.08 \pm 0.08$ & 4 & $75.48 \pm 0.08$ & $52.29 \pm 0.08$ & $83.27 \pm 0.02$ & $96.85 \pm 0.02$ & $84.19 \pm 0.08$ & 0.00 & 2 & 2 & 0.00 \\
\hline otadeceane & 42.093 & $\begin{array}{l}0.00 \\
2267.35 \pm 0 .\end{array}$ & $\begin{array}{l}0.00 \\
2266.85 \pm 0.0\end{array}$ & $\begin{array}{l}0.00 \\
4182.57 \pm 0 .\end{array}$ & $\begin{array}{l}0.00 \\
2068.87 \pm 0 .\end{array}$ & $\begin{array}{l}25.93 \pm 0.04 \\
1965.03 \pm 0 .\end{array}$ & $\begin{array}{l}0.00 \\
2682.77 \pm 0 .\end{array}$ & $\begin{array}{l}0.00 \\
2213.21 \pm 0 .\end{array}$ & $\begin{array}{l}0.00 \\
2544.96 \pm 0 .\end{array}$ & $\begin{array}{l}0.00 \\
88.15 \pm 0.0\end{array}$ & $\begin{array}{l}0.00 \\
4861.75 \pm 0 .\end{array}$ & 0.00 & $\begin{array}{l}0.00 \\
468.86 \pm 0 .\end{array}$ & $\begin{array}{l}0.00 \\
765.52 \pm 0 .\end{array}$ & $\begin{array}{l}0.00 \\
4603.22 \pm 0 .\end{array}$ & $\begin{array}{l}0.00 \\
1484.43 \pm 0 .\end{array}$ & $\begin{array}{l}0.00 \\
3102.32 \pm 0 .\end{array}$ & $\begin{array}{l}0.00 \\
3870.58 \pm 0 .\end{array}$ & $\begin{array}{l}0.00 \\
983.03 \pm 0.0\end{array}$ & $\begin{array}{l}96.05 \pm 0.02 \\
364+31 \pm \pm 0 .\end{array}$ & $\begin{array}{l}0.00 \\
679.15 \pm 0 .\end{array}$ & $\begin{array}{l}0.00 \\
3773.46 \pm 0 .\end{array}$ & $\begin{array}{l}0.00 \\
706.42 \pm 0 .\end{array}$ \\
\hline pentadceane & 30.888 & 05 & 3 & 01 & 08 & 06 & 07 & 05 & 06 & 3 & 05 & 0.00 & 04 & 04 & 08 & 08 & 08 & 08 & 2 & 08 & 02 & 02 & 08 \\
\hline phhtalic acid,isobutyl lester & 43.789 & 0.00 & 0.00 & 0.00 & $9.95 \pm 0.09$ & 0.00 & 0.00 & 0.00 & 0.00 & 0.00 & 0.00 & 0.00 & $\begin{array}{l}0.00 \\
102.72 \pm 0 .\end{array}$ & 0.00 & 0.00 & 0.00 & 0.00 & 0.00 & 0.00 & 0.00 & 0.00 & 0.00 & $\begin{array}{l}0.00 \\
136.27 \pm 0 .\end{array}$ \\
\hline  & 37.847 & 0.00 & 0.00 & 0.00 & 0.00 & 0.00 & 0.00 & 0.00 & 0.00 & 0.00 & $23.48 \pm 0.03$ & $26.57 \pm 0.04$ & 02 & 0.00 & $39.83 \pm 0.08$ & 0.00 & $13.04 \pm 0.08$ & $24.29 \pm 0.08$ & 0.00 & 40.44 & 108.84 & 20.61 & 08 \\
\hline
\end{tabular}




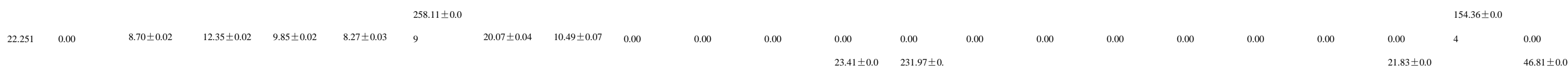



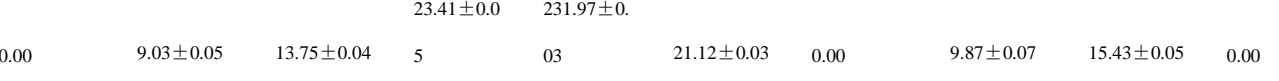



